\title{
PERHAPS SCALAR NEUTRINOS ARE THE LIGHTEST SUPERSYMMETRIC PARTNERS
}

\author{
John S HAGELIN \\ Department of Physics, Mahartsh International Universit, Fairfield IA 52556, USA \\ GL KANE* and S RABY ${ }^{* *}$ \\ Randall Laboratory of Phisics, University of Michigan, Ann Arbor, MI 48109, USA
}

Received 16 January 1984

\begin{abstract}
We consider the possibility that the scalar partners of the neutrinos $(\tilde{v})$ are the least massive supersymmetric partners, and show that this alternative is compatible with cosmological constraints, which put a significant lower bound on photino masses but not on $\tilde{y}$ masses Various consequences are examined the photon counting rate for $\mathrm{e}^{+} \mathrm{e}^{-} \rightarrow \gamma \tilde{p} \tilde{\tilde{p}}$ may be large, the rate for $\mathrm{e}^{+} \mathrm{e}^{-} \rightarrow \overline{\mathrm{W}}^{+} \tilde{\mathrm{W}}^{-}$by $\tilde{\nu}$ exchange is enhanced, $\mathrm{Z}^{0} \rightarrow \tilde{\nu} \overline{\tilde{\nu}}$ increases $\Gamma\left(\mathrm{Z}^{0}\right)$ by about $025 \mathrm{GeV}$, $\mathrm{W} \pm \rightarrow \tilde{\ell}^{ \pm} \tilde{v}$ may be enhanced, the decay $\tau \rightarrow \tilde{v}_{\tau} \ell \bar{\nu}_{\ell}$ may be detectable, there can be additional contributions to the rare decay $\mathrm{K}^{+} \rightarrow \pi^{+} \tilde{\nu} \overline{\tilde{\nu}}$, restrictions on gluno masses, which depend on photinos interacting before they decay, have to be re-examıned, scalar neutrinos have suitable charactenistics as candidates for dark matter in the universe We discuss one currently fashionable class of models that can predict a light $\tilde{v}$
\end{abstract}

There is presently great interest in theones with spontaneously broken supersymmetry [1], because of the hope such theories offer [2] for alleviating the hierarchy problem associated with the weak interaction scale. In such theories, supersymmetric partners of all the known light particles should exist with masses less than about 1 $\mathrm{TeV}$ if the desired technical improvement in the hierarchy problem is indeed to be obtained Thus, one way to test supersymmetric models experimentally is to search for those supersymmetric partners [3]. A number of recent analyses have explored the cosmological $[4,5]$ and terrestrial particle physics [6-9] implications of light gaugino-higgsino ("neutralıno") states. In this paper we explore the possibility that the scalar neutrino ("sneutrino") is the lightest supersymmetric partner

One astrophysical requirement for any supersymmetric theory with an exact $\mathrm{R}$ symmetry is that the lightest supersymmetric partner (LSP), which is stable against

\footnotetext{
* Research supported in part by US DOE

${ }^{\dagger}$ Present address T-8, MS B285, Los Alamos Natıonal Laboratory, Los Alamos, NM 87545, USA
} 
decay, can annihilate readily enough so that its present cosmological mass density is reduced to acceptably low levels Goldberg [4] has pointed out that due to a P-wave suppression in the annihilation of Majorana gauginos, this requirement leads to significant lower bounds on the photino mass if the photino is the lightest supersymmetric partner A recent and detaled analysis by Ellıs et al [5] places this lower bound at $\frac{1}{2} \mathrm{GeV}$ if the LSP is predominantly a photino or at $5 \mathrm{GeV}$ if the LSP is predomınantly a higgsino. Sneutrinos, by contrast, can pair-annıhılate via neutralıno exchange (fig 1a) without P-wave or helicity suppression, with no lower bound on their mass if they are the LSP

(1)

(a)

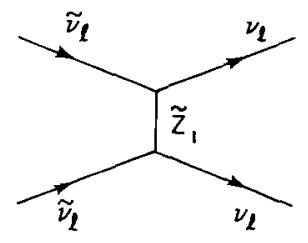

(b)

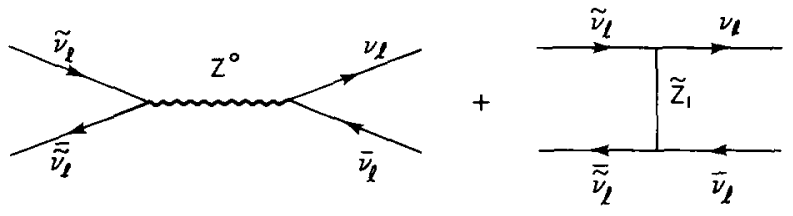

(c)
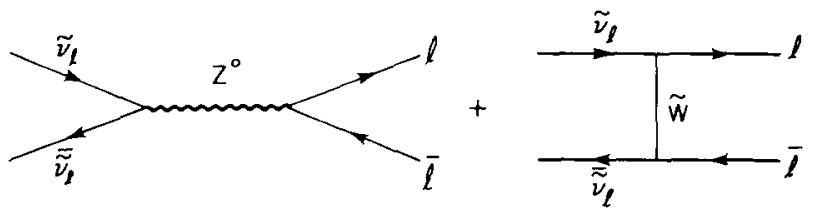

(d)

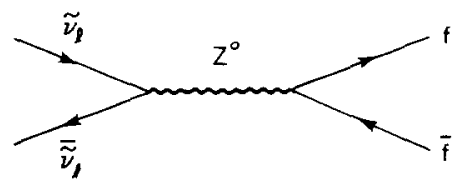

Fig 1 Dragrams contributing to sneutrino annhilation (a) $\tilde{\nu}_{\ell}+\tilde{\nu}_{\ell} \rightarrow \nu_{\ell}+\nu_{\ell}$, (b) $\tilde{\nu}_{\ell}+\overline{\tilde{\nu}}_{\ell} \rightarrow \nu_{\ell}+\bar{\nu}_{\ell}$, (c) $\tilde{\nu}_{\ell}+\overline{\tilde{\nu}}_{\ell} \rightarrow \ell+\bar{\ell}$, (d) $\tilde{\nu}_{\ell}+\overline{\bar{\nu}}_{\ell} \rightarrow \mathrm{f}+\overline{\mathrm{f}}, \mathrm{f} \neq \nu_{\ell}, \ell$ 
In the minimal supersymmetric extensions of the standard model, the cross sections for $\tilde{v}$ annihilation corresponding to figs la-d are

$$
\begin{aligned}
& \left(\sigma v_{\text {rel }}\right)_{1}=\frac{1}{16 \pi} \sum_{i=1}^{4}\left[\frac{\left(\alpha_{1} g_{2}-\beta_{i} g_{1}\right)^{2}}{M_{\tilde{z}_{1}}}\right]^{2}, \\
& \left(\sigma v_{\mathrm{rel}}\right)_{2}=\frac{s v_{\mathrm{rel}}^{2}}{192 \pi}\left[\frac{4 G_{\mathrm{F}}}{\sqrt{2}}+\sum_{i=1}^{4} \frac{\left(\alpha_{t} g_{2}-\beta_{i} g_{1}\right)^{2}}{2 M_{\tilde{\mathrm{z}}_{1}}^{2}}\right]^{2} \text {. } \\
& \left(\sigma v_{\mathrm{rel}}\right)_{3}=\frac{G_{\mathrm{F}}^{2} v_{\mathrm{rel}}^{2}}{4 \pi} \sqrt{1-\frac{4 m^{2}}{s}}\left[4 m^{2} \sin ^{2} \theta_{\mathrm{w}}\left(-\frac{1}{2}+\sin ^{2} \theta_{\mathrm{w}}\right)\right. \\
& \left.+\frac{2}{3}\left(\frac{1}{4}-\sin ^{2} \theta_{\mathrm{w}}+2 \sin ^{4} \theta_{\mathrm{w}}\right)\left(s-m^{2}\right)\right] \\
& +\frac{G_{\mathrm{F}}^{2} v_{\mathrm{rel}}^{2}}{2 \pi} \sqrt{1-\frac{4 m^{2}}{s}}\left[\frac{1}{3}\left(-\frac{1}{2}+\sin ^{2} \theta_{\mathrm{u}}\right)\left(m^{2}-s\right)-\sin ^{2} \theta_{\mathrm{u}} m^{2}\right] \\
& \times \sum_{i=1}^{2} \frac{O_{\tilde{u}_{i}}^{+2} M_{\mathrm{w}}^{2}}{M_{\tilde{\mathrm{u}}_{i}}^{2}} \\
& +\frac{G_{\mathrm{F}}^{2} v_{\mathrm{rel}}^{2}}{24 \pi} \sqrt{1-\frac{4 m^{2}}{s}}\left(s-m^{2}\right)\left[\sum_{i=1}^{2} \frac{O_{\tilde{\mathrm{w}}_{t}}^{+2} M_{\mathrm{u}}^{2}}{M_{\tilde{\mathrm{u}}_{t}}^{2}}\right]^{2} \\
& +\frac{G_{\mathrm{F}}^{2}}{2 \pi} m^{2}\left(1-\frac{4 m^{2}}{s}\right)^{3 / 2}\left[\sum_{i=1}^{2} \frac{O_{\tilde{u}_{1}}^{+2} M_{\mathrm{u}}^{2}}{M_{\dot{\mathrm{w}}_{t}}^{2}}\right]^{2}, \\
& \left(\sigma v_{\text {rel }}\right)_{4}=\frac{G_{\mathrm{F}}^{2} v_{\text {rel }}^{2}}{4 \pi} \sqrt{1-\frac{4 m^{2}}{s}}\left[-4 m^{2} x_{\mathrm{f}}\left(T_{3}^{\mathrm{f}}-x_{\mathrm{f}}\right)\right. \\
& \left.+\frac{2}{2}\left(T_{3}^{\mathrm{f}^{2}}-2 x_{\mathrm{f}} T_{3}^{\mathrm{f}}+2 x_{\mathrm{f}}^{2}\right)\left(s-m^{2}\right)\right]
\end{aligned}
$$

In eqs $(1 \mathrm{a}-\mathrm{d}) v_{\mathrm{rel}}$ is the relatıve velocity of the annihilatıng partıcles, $x_{\mathrm{f}} \equiv Q_{\mathrm{f}} \sin ^{2} \theta_{\mathrm{w}}$ and " $m$ " always denotes the final state fermion mass Since the sneutrinos annihilate non-relativistically, higher powers in $v_{\text {rel }}$ are not maintained. The quantities $M_{\bar{z}_{i}}$ are the eigenvalues corresponding to the four "neutralıno" mass eigenstates

$$
\tilde{Z}_{l} \equiv \alpha_{\imath} \tilde{W}^{3}+\beta_{\imath} \tilde{B}+\gamma_{1} \tilde{H}_{1}^{0}+\delta_{l} \tilde{H}_{2}^{0} \quad(l=1 \quad 4),
$$


of the Majorana mass matrux $[5,7,8]$

$$
\left(\tilde{W}^{3}, \tilde{B}, \tilde{H}_{1}^{0}, \tilde{H}_{2}^{0}\right)\left(\begin{array}{cccc}
M_{2} & 0 & -\sqrt{\frac{1}{2}} g_{2} v_{1} & \sqrt{\frac{1}{2}} g_{2} v_{2} \\
0 & M_{1} & \sqrt{\frac{1}{2}} g_{1} v_{1} & -\sqrt{\frac{1}{2}} g_{1} v_{2} \\
-\sqrt{\frac{1}{2}} g_{2} v_{1} & \sqrt{\frac{1}{2}} g_{1} v_{1} & 0 & \varepsilon \\
\sqrt{\frac{1}{2}} g_{2} v_{2} & -\sqrt{\frac{1}{2}} g_{1} v_{2} & \varepsilon & 0
\end{array}\right)\left(\begin{array}{c}
\tilde{W}_{3} \\
\tilde{B} \\
\tilde{H}_{1}^{0} \\
\tilde{H}_{2}^{0}
\end{array}\right)
$$

arising from the Higgs vacuum expectation values $v_{12} \equiv\left\langle 0\left|H_{1,2}^{0}\right| 0\right\rangle$ and the lagrangian terms

$$
\varrho \supset \varepsilon \varepsilon_{\alpha \beta} \tilde{H}_{1}^{\alpha} \tilde{H}_{2}^{\beta}-M_{2} \tilde{W}^{a} \tilde{W}^{a}-M_{1} \tilde{B} \tilde{B}
$$

$\tilde{W}^{a}$ and $\tilde{B}$ are $\mathrm{SU}(2)$ and $\mathrm{U}(1)$ gaugınos, and $\alpha, \beta(a)$ are doublet (triplet) $\mathrm{SU}(2)$ indices. For numerical purposes we shall assume $M_{1}=\left(5 \alpha_{1} / 3 \alpha_{2}\right) M_{2}$ where $\alpha_{12} \equiv$ $g_{1,2}^{2} / 4 \pi$ are the $S U(2)$ and $U(1)$ couplings, which holds to leading order in the renormalization group equations if weak $S U(2) \times U(1)$ is eventually embedded in a unifying non-abelian group The quantities $M_{\tilde{\mathrm{w}}_{\text {, }}}$ which appear in eq (1c) are the elgenvalues of the charged gaugıno-higgsino mass matrix $[5,7,8]$

$$
\left(\tilde{W}^{+}, \tilde{H}_{1}^{+}\right)_{L}\left(\begin{array}{cc}
M_{2} & g_{2} v_{2} \\
g_{2} v_{1} & -\varepsilon
\end{array}\right)\left(\begin{array}{c}
\tilde{W}^{-} \\
\tilde{H}_{2}^{-}
\end{array}\right)_{\mathrm{L}}
$$

and the quantities $O_{\tilde{w}_{i}}^{+}$represent the coefficient of $\tilde{W}_{\mathrm{L}}^{+}$in the $i$ th charged eigenstate - the cosine or sine of the angle $\theta_{+}$which rotates the positively charged chiral fields in eq (5).

To compute the cosmological mass density of the sneutrino $\left(\rho_{\bar{\nu}}\right)$ we recall the rate equation for the number density of annihilating particles [10]

$$
\frac{\mathrm{d} n}{\mathrm{~d} t}=-3 \frac{R}{R} n-\left\langle\sigma v_{\mathrm{rel}}\right\rangle\left(n^{2}-n_{0}^{2}\right),
$$

where $n$ is the actual number density at time $t, n_{0}$ is the number density of sneutrinos in thermal equilibrium, $R$ is the cosmic scale factor, and angular brackets denote thermal average Following standard methods [10] one can rewrite (6) in the convenient form

$$
\frac{\mathrm{d} f}{\mathrm{~d} x}=\frac{M_{\tilde{\nu}}}{k^{3}}\left(\frac{8 \pi^{3} N_{\mathrm{F}} G}{45}\right)^{-1 / 2}\left\langle\boldsymbol{\sigma} v_{\mathrm{rel}}\right\rangle\left(f^{2}-f_{0}^{2}\right)
$$

where $x \equiv k T / M_{\tilde{\nu}}, f(x)=n / T^{3}, f_{0}(x)=n_{0} / T^{3}, k$ is the Boltzmann constant, $G=1 / M_{\mathrm{pi}}^{2}$ is Newton's constant and $N_{\mathrm{F}}$ counts the effective number of degrees of freedom at a given temperature 
Replacing $v_{\text {rel }}$ in eqs. $(1 \mathrm{a}-\mathrm{d})$ by its thermal average $\left(v_{\text {rel }}^{2} \simeq 6 k T / M_{\tilde{v}}=6 x\right)$. the cross section can be written

$$
\left\langle\sigma v_{\text {rel }}\right\rangle=\tilde{a}+\tilde{b} x
$$

and the rate eq. (7) takes the general form

$$
\begin{aligned}
\frac{\mathrm{d} f}{\mathrm{~d} x} & =\frac{M_{\tilde{v}}}{k^{3}}\left(\frac{8 \pi^{3} N_{\mathrm{F}} G}{45}\right)^{-1 / 2}(\tilde{a}+\tilde{b} x)\left(f^{2}-f_{0}^{2}\right) \\
& \equiv(a+b x)\left(f^{2}-f_{0}^{2}\right)
\end{aligned}
$$

Following the analytic approximation of Lee and Weinberg [10], we expect the scaled number density $f(x)$ in eq. (9) to remain approximately equal to its equilibrium value $f_{0}(x)$ until the temperature $T$ drops to a freeze-out value of $T_{\mathrm{f}}$ where the annihilation rate is equal to the rate of change in $f_{0}$

$$
\frac{\mathrm{d} f_{0}}{\mathrm{~d} x}=(a+b x) f_{0}^{2} \quad \text { at } \quad x=x_{\mathrm{f}},
$$

and assume that thereafter it evolves approximately according to the equation

$$
\frac{\mathrm{d} f}{\mathrm{~d} x}=(a+b x) f^{2},
$$

subject to the initial conditions $f\left(x_{\mathrm{f}}\right)=f_{0}\left(x_{\mathrm{f}}\right)$ Since $x_{\mathrm{f}} \ll 1$ for the sneutrino, we can use the non-relativistic approximation [10]

$$
f_{0}(x)=2 k^{3}(2 \pi x)^{-3 / 2} \mathrm{e}^{-1 / x}
$$

to solve for the freeze-out temperature Eqs (10) and (12) together give

$$
\begin{aligned}
x_{\mathrm{f}} & =\frac{1}{\ln \left(a x_{\mathrm{f}}^{1 / 2}+b x_{\mathrm{f}}^{3 / 2}\right)+\ln \left[2 k^{3} /(2 \pi)^{3 / 2}\right]} \\
& =\frac{1}{\ln \left(\tilde{a} x_{\mathrm{f}}^{1 / 2}+\tilde{b} x_{\mathrm{f}}^{3 / 2}\right)-\frac{1}{2} \ln \left(16 \pi^{6} N_{\mathrm{F}} G / 45 M_{\tilde{v}}^{2}\right)}
\end{aligned}
$$

The present number density is obtained by integrating eq (12) from $x=x_{\mathrm{f}}$ down to $x=0$

$$
f(0)=\frac{1}{a x_{\mathrm{f}}+\frac{1}{2} b x_{\mathrm{f}}^{2}}
$$


The present mass density $\rho_{\tilde{\nu}}$ is then simply

$$
\rho_{\bar{\nu}}=08\left(\frac{T_{\hat{\nu}}}{T_{\gamma}}\right)^{3} T_{\gamma}^{3} M_{\bar{\nu}} \frac{1}{a x_{\mathrm{f}}+\frac{1}{2} b x_{\mathrm{f}}^{2}},
$$

where $\left(T_{\tilde{v}} / T_{\gamma}\right)^{3}$ accounts for the subsequent reheatıng of the photon temperature with respect to the temperature of $\tilde{\nu}$, due to the annihilation of particles with $M<x_{\mathrm{f}} M_{\bar{\nu}}$, and is tabulated [11] together with $N_{\mathrm{F}}$ in table 1. The "fudge factor" 08 is included to correct for the fact [10] that the analytic approximation (10), (11) to the full rate eq (7) gives a result which is approximately $25 \%$ too large. In terms of the coefficients $\tilde{a}, \tilde{b}$ appearing in the annihilation cross section, the mass density (15) reads

$$
\rho_{\bar{\nu}}=50 \times 10^{-40}\left(T_{\gamma} / 28^{\circ} \mathrm{K}\right)^{3}\left(\frac{43}{22 N_{\mathrm{F}}^{1 / 2}}\right) \frac{1}{\tilde{a} x_{\mathrm{f}}+\frac{1}{2} \tilde{b} x_{\mathrm{f}}^{2}} \mathrm{~g} / \mathrm{cm}^{3} .
$$

It is now straightforward to incorporate into eq. (16) the results of a numerical analysis of the cross section (1a-d) (including the diagonalization of the mass matrices (3) and (5)) and to compare the present mass density $\rho_{i}$ with its cosmological upper bound We know from the rate of expansion of the universe that $\rho_{\tilde{v}} \leqslant 2 \times 10^{-29}\left(\Omega h_{0}^{2}\right) \mathrm{g} / \mathrm{cm}^{3}$, where $\Omega$ is the density in units of the closure density

TABLE 1

$\left(T_{\bar{v}} / T_{\gamma}\right)^{3}$ accounts for the subsequent reheating of the photon temperdure with respect to the $\vec{v}$ temperature due to the annihilation of particles with $M<x_{\mathrm{f}} M_{i} \quad N_{\mathrm{F}}$ counts the effective number of degrees of freedom at a given temperature

\begin{tabular}{ccc}
\hline$T_{\mathrm{f}}$ & $N_{\mathrm{f}}$ & $\left(T_{i} / T_{\gamma}\right)^{3}$ \\
\hline$m_{\mathrm{e}}-m_{\mu}$ & $\frac{43}{8}$ & 275 \\
$m_{\mu}-m_{\pi}$ & 57 & 365 \\
$m_{\pi}-T_{\mathrm{H}}$ & $\frac{69}{8}$ & 441 \\
$T_{\mathrm{H}}-m_{\mathrm{s}}$ & $\frac{205}{8}$ & 131 \\
$m_{\mathrm{s}}-m_{\mathrm{c}}$ & $\frac{247}{8}$ & 158 \\
$m_{\mathrm{c}}-m_{\tau}$ & $\frac{2 \times 9}{8}$ & 185 \\
$m_{\tau}-m_{\mathrm{b}}$ & $\frac{303}{8}$ & 194 \\
$m_{\mathrm{b}}-m_{\mathrm{t}}$ & $\frac{345}{8}$ & 221 \\
$m_{\mathrm{t}}-M_{\mathrm{W}}$ & $\frac{3 \times 7}{8}$ & 248 \\
$>M_{\mathrm{W}}$ & $\frac{423}{8}$ & 271 \\
\hline
\end{tabular}

This table is adapted from ref [11] Most of the notation is described in the text, with the exception that $T_{\mathrm{H}}$ is the temperature above which it is supposed that hadrons should be described in term of quark and gluon degrees of freedom 
and $h_{0}$ is the Hubble parameter in units of $100 \mathrm{~km} \mathrm{~s}^{-1} \mathrm{Mpc}^{-1}$ It is reasonable to believe that $\Omega h_{0}^{2} \leqslant 1$ implying that $\rho_{\tilde{v}} \leqslant 2 \times 10^{-29} \mathrm{~g} / \mathrm{cm}^{3}$

Fig la gives a large contribution to the annihilation cross section (1a) which is independent of $s, v_{\text {rel }}$ and hence $M_{\tilde{v}}$. Physically this is because a Majorana neutralıno can be exchanged, with an amplitude going as $1 / M_{\tilde{z}_{l}}$ (rather than $1 / M_{\tilde{z}_{1}}^{2}$ ), so $\sigma \sim 1 / M_{\tilde{z}_{i}}^{2}$ and no dependence on $M_{\tilde{\nu}}$ enters It is therefore apparent from eq (16) that $\rho_{\tilde{\nu}}$ is approximately independent of $M_{\tilde{\nu}}$. This contrasts with the familiar cases where the LSP is a Majorana neutralino [4.5] or even a heavy neutrino [10], where the annihilation cross section is proportional to $M^{2}$ (or to a final state mass ${ }^{2}$ ). In such cases, the cosmological mass density grows at least as $1 / M^{2}$ for small $M$, which leads to a lower bound on $M$ In the present case, however, there is no lower bound on $M_{\tilde{v}}$ provided the contribution to the cross section from fig la is non-negligible ${ }^{\star}$

In order for the contribution to $\left\langle\sigma v_{\text {rel }}\right\rangle$ from fig. 1a to be significant, it is necessary that the gaugino or higgsino mass terms $M_{2}, \varepsilon$ in the lagrangian (4) be non-negligible In the limit of $M_{2}, \varepsilon \rightarrow 0$, there is a light photıno and a light higgsino elgenstate

$$
\begin{aligned}
\tilde{\gamma} \equiv \frac{g_{1} \tilde{W}^{3}+g_{2} \tilde{B}}{\sqrt{g_{1}^{2}+g_{2}^{2}}}, & M_{\tilde{\gamma}} \approx \frac{8}{3} \frac{g_{1}^{2}}{g_{1}^{2}+g_{2}^{2}} M_{2}, \\
\tilde{S}^{0} \equiv \frac{v_{2} \tilde{H}_{1}^{0}+v_{1} \tilde{H}_{2}^{0}}{v}, & M_{\tilde{\mathrm{S}}} \approx \frac{2 v_{1} v_{2}}{v^{2}} \varepsilon,
\end{aligned}
$$

where we have introduced $v \equiv \sqrt{v_{1}^{2}+v_{2}^{2}}$ In this same limit the remaining two eigenstates are

$$
\tilde{Z}_{ \pm} \equiv \frac{g_{1} \tilde{B}-g_{2} \tilde{W}^{3} \pm \sqrt{g_{1}^{2}+g_{2}^{2}}\left(v_{1} \tilde{H}_{1}^{0}-v_{2} \tilde{H}_{2}^{0}\right) / v}{\sqrt{2\left(g_{1}^{2}+g_{2}^{2}\right)}}, \quad M_{\tilde{Z}_{1}} \simeq M_{Z}=\sqrt{\frac{1}{2}\left(g_{1}^{2}+g_{2}^{2}\right)} v
$$

Neither the photino $(\tilde{\gamma})$ nor the higgsino $\left(\tilde{\mathrm{S}}^{0}\right)$ contributes to sneutrino annihilation, while the two degenerate $\tilde{Z}_{ \pm}$neutralınos give contributions that are equal and opposite and hence cancel However if the higgsino mass parameter $\varepsilon$ is $\gtrsim O(1$ $\mathrm{GeV}$ ), this splits the degeneracy between the two $\tilde{Z}_{ \pm}$eigenstates enough so that the sneutrinos annihilate easily, in which case there is no lower bound on the $\tilde{v}$ mass from cosmology (see previous footnote) (In contrast to fig 1a which is "semi-weak," figs. $1 \mathrm{~b}$-d give weak contributions to the $\tilde{v}$ annihilation cross sections which also

* There is a separate constraint [11] that the sneutrino be non-relativistic dunng the time of helium synthesis Otherwise the expansion rate which depend on the total energy density and therefore on the number of relativistic particles present, would be too fast, causing the weak interactions to freeze out at a higher temperature resulting in more neutrons and a higher concentration of primordial ${ }^{4} \mathrm{He}$ This allow us to conclude that $M_{\bar{i}} \geqslant$ few $\mathrm{MeV}$ 
suffer from helicity and/or P-wave suppression. In the absence of fig. 1a, figs. 1b-d would lead to a lower bound on $M_{\tilde{v}}$ which is comparable to those derived for the photmo as LSP ) It therefore follows that by varying the parameters in the lagrangian (4), particularly $\varepsilon$, one can vary the strength of fig $1 \mathrm{a}$ and thereby adjust the present mass density of $\tilde{\nu}$ to any desired value up to or exceeding closure density This makes the sneutrıno a potentrally interestıng dark matter candıdate

There is considerable evidence that the dominant form of energy in the universe is neither luminous nor baryonic [12] This appears to be true on mass scales ranging from that of dwarf spheroidal galaxies $\left(\sim 10^{7} \mathrm{M}_{\odot}\right)$ to rich clusters of galaxies $\left(\sim 10^{15} M_{\bigcirc}\right.$ where $\left.M_{\odot} \sim 2 \times 10^{33} \mathrm{~g}\right)$ [12] Several elementary particles have been proposed as candidates for this dark matter. They typically lead to one of three distinct scenarios for galaxy formation. hot, warm or cold matter (see the review by Prımack and Blumenthal [12]) Scalar neutrinos fall into the category of cold matter, thus joining the list of candidates which includes axions, photinos and massive gravitinos We briefly outline this scenario.

Let us define the temperature $\left(T_{\text {eq }}\right)$ when the radiation energy density $\left(\rho_{\mathrm{r}}\right)$ equals the sneutrino energy density $\left(\rho_{\bar{v}}\right)$ For temperatures $T>T_{\text {eq }}$ the universe is radiation dominated We have

$$
T_{\mathrm{eq}} \cong 5 \Omega_{\tilde{\nu}} h_{0}^{2} \quad \mathrm{eV},
$$

where $h_{0}$ (defined previously) satısfies $05 \leqslant h_{0} \leqslant 1$ and $\Omega_{\bar{v}} \equiv \rho_{\bar{v}} / \rho_{\mathrm{c}}$ is the sneutrino energy density today in units of the closure density

$$
\rho_{\llcorner}=\frac{3 H_{0}^{2}}{8 \pi G}=188 \times 10^{-29} h_{0}^{2} \quad \mathrm{~g} / \mathrm{cm}^{3}
$$

Initially at some temperature $T \gg T_{\text {eq }}$ sneutrinos are in thermal equilibrium with radiation However at the temperature $T_{\mathrm{f}} \gg T_{\text {eq }}$, sneutrinos decouple, going out of both kinetic and chemical equilibrium. Typically $T_{\mathrm{f}} \sim \frac{1}{20} M_{\tilde{v}}$ and sneutrinos are non-relativistic at this time.

Proor to decoupling, sneutrınos are prevented from clusterıng by radiation pressure. Density fluctuations which enter the horizon at this time oscillate acoustically However, for $T<T_{\mathrm{f}}$ density fluctuations within the horizon can grow During the epoch $T_{\text {eq }}<T<T_{\mathrm{f}}$ the clusterıng tıme scale $\left(\sim\left(G \rho_{\bar{v}}\right)^{-1 / 2}\right)$ is much longer than the expansion time $\left(\sim\left(G \rho_{\mathrm{r}}\right)^{-1 / 2}\right)$ As a result fluctuations inside the horizon grow very slowly according to the relation [13]

$$
\delta_{\tilde{\nu}} \equiv \frac{\delta \rho_{\bar{v}}}{\rho_{\bar{\nu}}}=\left.\delta_{\bar{\nu}}\right|_{t}\left(1+\frac{3}{2} \frac{\rho_{i \bar{\nu}}(T)}{\rho_{\mathrm{r}}(T)}\right)
$$

which is valıd for scales greater than the free streaming mass $\left(M_{\mathrm{FS}}\right)$ for sneutrinos 
$\left(\left.\delta_{\bar{v}}\right|_{t}\right.$ is the initial fluctuation spectrum at the time it enters the horizon Its origin is unclear. One possibility is that the largest contribution is imposed on sneutrinos by the dominant radiation background. If this is the case, then sneutrino fluctuatıons outsıde the horizon are given by [14]

$$
\left.\delta_{\hat{\nu}}\right|_{\imath}=\frac{3}{4} \delta_{\mathrm{r}}
$$

In some recent scenarıos based on an "inflationary" universe [15], it may naturally have the Zeldovich spectrum [16]

$$
\delta_{\tilde{\nu}} \sim 10^{-4} \quad \text { (independent of scale) }
$$

as it enters the horizon.)

The free streaming mass $\left(M_{\mathrm{FS}}\right)$ [17] is defined by the mass of sneutrinos in a volume determined by the distance $\left(d_{\mathrm{FS}}\right)$ sneutrinos can travel in a Hubble expansion time

We have

$$
\begin{gathered}
d_{\mathrm{FS}} \simeq\left(\frac{\left\langle\boldsymbol{v}^{2}\right\rangle}{G \rho_{\mathrm{r}}(T)}\right)^{1 / 2}, \\
M_{\mathrm{FS}}(T)=\frac{4}{3} \pi d_{\mathrm{FS}}^{3}(T) \rho_{\tilde{\nu}}(T) .
\end{gathered}
$$

At $T=T_{\text {eq }}$

$$
\left.M_{\mathrm{FS}}\right|_{\mathrm{eq}} \simeq 10^{-5} \mathrm{M}_{\mathrm{O}}\left(\frac{M_{\tilde{v}}}{1 \mathrm{GeV}}\right)^{-3} \Omega_{\tilde{\nu}} h_{0}^{2}
$$

where we have taken $T_{\mathrm{f}}=\frac{1}{20} M_{\tilde{\nu}}$ This is to be compared to the mass of sneutrinos within the horizon at $T_{\text {eq }}$.

$$
\left.M_{\mathrm{H}}\right|_{\mathrm{eq}} \simeq 10^{16} \mathrm{M}_{\mathrm{O}} h_{0}^{-4} \Omega_{\tilde{v}}^{-1 / 2}
$$

It is these fluctuations on scales $M$ for $M_{\mathrm{FS}} \leqslant M \leqslant M_{\mathrm{H}}$ which are the first to begin growing at $T<T_{\text {eq }}$. They grow according to the relation [18]

$$
\left.\delta_{\tilde{\nu}}\right|_{T<T_{\text {eq }}}=\left.\delta_{\tilde{\nu}}\right|_{\mathrm{eq}}\left(\frac{T_{\mathrm{eq}}}{T}\right)
$$

Given $T_{\text {eq }} / T_{0} \sim 24 \times 10^{4} \Omega_{\bar{\nu}} h_{0}^{2}$, we see that initial fluctuations $\delta_{\grave{\nu}} l_{\text {, }}$ of order $10^{-4} \mathrm{can}$ certainly become nonlinear by the present epoch (Note in hot or warm scenarios of dark matter, the fluctuations on scales of order $10^{15} \mathrm{M}_{\odot}$ or $10^{12} \mathrm{M}_{\circ}$ (resp) are the first to grow It is difficult, in these scenarios, to explain the presence of dark matter on small scales of order $10^{7} \mathrm{M}_{\circ}$.) 
Baryons do not begin to cluster until after the temperature of hydrogen recombination $\left(T_{\text {rec }} \sim 0.3 \mathrm{eV}\right)$ They can then cluster about the dominant sneutrino background In the perturbative regime we have [19]

$$
\delta_{\mathrm{b}}+2 H \delta_{\mathrm{b}}=4 \pi G \rho_{\bar{v}} \delta_{\bar{\nu}}
$$

One finds that in a Hubble expansion time $\delta_{\mathrm{b}} \simeq \delta_{\bar{v}}$ This equation is valid for baryons within the horizon and on scales greater than the baryon free streaming mass ( $M_{\mathrm{FSb}} \simeq 10^{5} \mathrm{M}_{\bigcirc}$ at recombination)

We thus have a picture of fluctuations on all scales from stars to clusters of galaxies becoming nonlınear at about the same epoch

It is not yet clear whether the large-scale structure of the universe can be explained in such a scenario. Recent computer simulations by Melott et al [20] are, however, encouraging.

Finally we note that if sneutrinos are relevant as dark matter candidates, we can place a rough upper limit on their mass This is because the sneutrino energy density today (or equivalently $\Omega_{i j}$ ) is (as we discussed earlier) very sensitive to the higgsino mass parameter $\varepsilon$ If we demand that sneutrinos are the LSP and that $\Omega_{\tilde{v}} \sim 01$ we find $M_{\tilde{v}} \leqslant 10 \mathrm{GeV}$. In order to increase $\Omega_{\tilde{\nu}}$ we must decrease $\varepsilon$ and hence $M_{\tilde{v}}$ For example $\Omega_{\tilde{\nu}} \sim 025$ imples $M_{\tilde{v}} \leq 5 \mathrm{GeV}$ and $\Omega_{\tilde{\nu}} \sim 1$ imples $M_{\tilde{\nu}} \leq 2 \mathrm{GeV}$

Before we consider the various implications for particle physics of a light scalar neutrıno, we will discuss the kınds of supersymmetric models which could predict a sneutrino for the LSP

In the minimal low energy supergravity model (MLES) [21] the sneutrino mass (for all three families) is given by the expression ${ }^{\star}$

$$
M_{\tilde{\nu}}^{2}=m_{\mathrm{g}}^{2}-\frac{1}{2} M_{2}^{2}\left(\frac{v_{1}^{2}-v_{2}^{2}}{v^{2}}\right),
$$

where $m_{\mathrm{g}}$ is the gravitino mass and $v_{1} \geq v_{2}$. Clearly sneutrinos are lighter than gravitınos. Gaugino and higgsino masses depend on the Majorana mass parameters $M_{3}, M_{2}, M_{1}$ for $\mathrm{SU}_{3}, \mathrm{SU}_{2}, \mathrm{U}_{1}$ respectively and the Higgs mass parameter $\varepsilon$ (eq (4)) It is always possible to choose these parameters such that sneutrinos are lighter than all gauginos and higgsinos.

Let us now consider squark and slepton masses For the latter we derive a simple constraint such that $M_{\tilde{v}}<M_{\hat{\ell}}$. The slepton mass matrix is given by

$$
M_{\tilde{\ell}}^{2}=\left(\begin{array}{cc}
m_{\mathrm{g}}^{2}+m_{\ell}^{2}+\frac{1}{4}\left(g_{2}^{2}-g_{1}^{2}\right)\left(v_{1}^{2}-v_{2}^{2}\right) & A m_{\mathrm{g}} m_{\ell} \\
A m_{\mathrm{g}} m_{\ell} & m_{\mathrm{g}}^{2}+m_{\ell}^{2}+\frac{1}{2} g_{1}^{2}\left(v_{1}^{2}-v_{2}^{2}\right)
\end{array}\right)
$$

* In the following analysis we have ignored corrections coming from gauginos These corrections for sleptons are proportional to $\alpha_{1}, \alpha_{2}$ and the gaugino mass terms $M_{1}, M_{2}$ For $M_{1} M_{2} \leq m_{\mathrm{g}}$, these corrections are negligible 
where $m_{\ell}$ is the corresponding lepton mass and $A$ is an arbitrary parameter, typically of order 1 We find $M_{\bar{v}}>M_{\tilde{\ell}}$ if

$$
\frac{M_{\mathrm{u}}^{2}}{m_{\mathrm{g}} m_{\ell}}\left(\frac{1+3 \tan ^{2} \theta_{\mathrm{w}}}{2}\right)^{1 / 2}\left(\frac{v_{1}^{2}-v_{2}^{2}}{v^{2}}\right)>A
$$

This constraint is easily satisfied For example even if $\left(v_{1}^{2}-r_{2}^{2}\right) / v^{2}$ is as small as 014 and $m_{\ell}=m_{\tau}$ we find the constraint

$$
\left(\frac{m_{\mathrm{g}}}{1 \mathrm{GeV}}\right) A<520
$$

The situation regarding squarks is more complicated If $M_{3}-m_{\mathrm{g}}$ then squarks obtain significant renormalization group corrections to their tree-level masses These tend to increase the squark to slepton mass ratio In addition for third generation squarks, large Yukawa couplings also affect the runnıng masses There is one range of parameters where we can simply analyze the squark spectrum, 1 e $A \ll 1$ and $M_{3} \ll m_{\mathrm{g}}$ In this limit the sneutrino is lighter than all squarks [22]

We conclude that there is certainly a range of parameter space in the MLES model for which sneutrinos are the LSP

At what scale might we expect $M_{\vec{\nu}}$ From eq (26) we see that $M_{\tilde{v}}$ can be as small as $\sim \frac{1}{10} m_{\mathrm{g}}$ without extreme fine tunıng. We could thus reasonably expect $M_{\bar{\nu}}$ to be of order $1-10 \mathrm{GeV}$.

We now would like to make some phenomenological remarks about the sneutrınos as LSP, beginning with the photon counting experiment $\mathrm{e}^{+} \mathrm{e}^{-} \rightarrow \gamma \tilde{\nu} \tilde{\tilde{\nu}}$ The $\tilde{\boldsymbol{\nu}} \tilde{\tilde{\nu}}$ production cross section due to $Z$ exchange is simply half of the conventional neutrino production cross section neglectıng phase space [23]

$$
\frac{\Gamma\left(\mathrm{e}^{+} \mathrm{e}^{-\stackrel{Z}{\rightarrow} \tilde{\nu} \overline{\tilde{\nu}}}\right)}{\Gamma\left(\mathrm{e}^{+} \mathrm{e}^{-\stackrel{z}{\rightarrow} \nu \bar{\nu})}\right.}=\frac{1}{2}\left(1-4 M_{\bar{\nu}}^{2} / s\right)^{3 / 2}
$$

However, the electron-sneutrino recelves an extra contribution from $\tilde{W} \pm$ exchange which can be larger than the $\mathrm{W}^{ \pm}$contribution to the electron-neutrino cross section by the ratio $\left(M_{\mathrm{W}} / M_{\tilde{\mathrm{W}}}\right)^{4}$ Since there are in fact two charged mass eigenstates (5) which contribute to sneutrino production through their $\tilde{W}^{ \pm}$components, the exact cross section is quite model dependent.

$$
\begin{aligned}
& \sigma\left(\mathrm{e}^{+} \mathrm{e}^{-} \rightarrow \tilde{\nu} \overline{\tilde{\nu}}\right)=\frac{G_{\mathrm{F}}^{2} s \beta^{3 / 2}}{12 \pi}\left\{\frac{1}{4}-\sin ^{2} \theta_{w}+2 \sin ^{4} \theta_{\mathrm{w}}+\left(\frac{1}{2}-\sin ^{2} \theta_{\mathrm{u}}\right) \sum_{t=1}^{2}\left(O_{\tilde{w}_{i}}^{+2} \frac{M_{\mathrm{w}}^{2}}{M_{\tilde{\mathrm{w}}_{t}}^{2}}\right)\right. \\
&\left.+\frac{1}{4} \sum_{t=1}^{2}\left(O_{\overline{\mathrm{w}}_{t}}^{+2} \frac{M_{\mathrm{w}}^{2}}{M_{\tilde{\mathrm{u}}_{i}}^{2}}\right)^{2}\right\}
\end{aligned}
$$


where $\beta=1-4 M_{\tilde{\nu}}^{2} / s$ and the last two terms in brackets only contribute for $\tilde{\nu}_{\mathrm{e}}$ production. In the limit of $M_{2}, \varepsilon \rightarrow 0$, the charged gaugino-higgsino mass eigenstates become the Dirac fermions $\left(\tilde{\mathrm{H}}_{2}^{-}, \tilde{\mathrm{W}}^{+}\right)$and $\left(\tilde{\mathrm{W}}^{-}, \tilde{\mathrm{H}}_{1}^{+}\right)$with masses $g_{2} v_{2}$ and $g_{2} v_{1}$, respectively It is the first of these, which is also presumably the lighter if $v_{1}>v_{2}$ as expected, that contributes to $\mathrm{e}^{+} \mathrm{e}^{-} \rightarrow \tilde{\nu} \overline{\tilde{\nu}}$ so it is quite plausible that the cross section could be substantial Note. for $\varepsilon$ small ( $1 \mathrm{e}$ the cosmologically interesting regime where $\Omega_{\hat{v}} \simeq\left(\frac{1}{10}-1\right)$ ) and $v_{1} \geq 4 v_{2}$, the cross section for $\tilde{\nu}_{\mathrm{e}}$ production is roughly 100 times greater than for $\tilde{\nu}_{\mu}$ or $\tilde{\nu}_{\tau}$ production This is because the lightest charged wino eigenstate has mass $\leqslant 30 \mathrm{GeV}$ in this regime. The radiatıve productıon cross section is simply related to the bare process in the interesting "soft" photon limit

$$
\frac{\mathrm{d}^{2} \sigma\left(\mathrm{e}^{+} \mathrm{e}^{-} \rightarrow \gamma \tilde{\nu} \tilde{\tilde{\nu}}\right)}{\mathrm{d} x_{\gamma} \mathrm{d}\left(\cos \theta_{\gamma}\right)} \approx \frac{2 \alpha}{\pi} \frac{\sigma\left(\mathrm{e}^{+} \mathrm{e}^{-} \rightarrow \tilde{\nu} \tilde{\tilde{\nu}}\right)}{x_{\gamma} \sin ^{2} \theta_{\gamma}}
$$

where $x_{\gamma} \equiv 2 E_{\gamma} / E_{\mathrm{cm}}$. A signal for $\mathrm{e}^{+} \mathrm{e}^{-} \rightarrow \gamma+$ "unobserved" above the standard model prediction can be interpreted as additional neutrino species or as evidence for photinos or sneutrinos

It is also worth noting that if the $\tilde{\nu}$ is light, the cross section for $\mathrm{e}^{+} \mathrm{e}^{-} \rightarrow \tilde{\mathrm{W}}^{+} \tilde{\mathrm{W}}^{-}$by $\tilde{\nu}$ exchange is enhanced, so that winos would be easier to observe up to the kinematic lımit for producing them

If $M_{\hat{\nu}} \leq \mathrm{O}(10) \mathrm{GeV}$ there is no significant phase space suppression for its contribution to $\mathrm{Z}$ decay Since a light $\tilde{\nu}$ contributes half as much as a conventional $\nu$ flavor, the $\tilde{\nu}+\overline{\tilde{\nu}}$ contribution is $\frac{1}{2}$ of the standard model $\nu$ prediction The latter is $6 \%$ per flavor, or $18 \%$ for three flavors, so the $\tilde{\nu}$ contribution is $9 \%$ for three flavors The expected $Z$ width is then 109 times the standard model prediction, which is an increase of about $025 \mathrm{GeV}$, just due to $\tilde{v}$ contributions

Note that while photmos and sneutrinos both contribute to the photon counting experıment $\mathrm{e}^{+} \mathrm{e}^{-} \rightarrow \gamma+$ "unobserved" at low energies, the photıno does not contribute to the $Z$ width [8]. (Conversely, a light higgsino would contribute to the $Z$ width but would not contribute significantly to $\mathrm{e}^{+} \mathrm{e}^{-} \rightarrow \gamma+$ "unobserved" at low energies [8]) Thus $Z$ decay and photon countıng at $\sqrt{s} \approx 30 \mathrm{GeV}$ provide complimentary information on supersymmetric particles

If $M_{\bar{\nu}} \leqslant 1 \mathrm{GeV}$, the decay $\tau \rightarrow \tilde{\nu}_{\tau} \ell \overline{\tilde{\nu}}_{\ell}\left(\ell=\mu\right.$ or e) becomes possible through $\tilde{\mathrm{W}}^{ \pm}$ exchange The implications of the non-observation of this decay have been studied in ref [24] If $M_{\tilde{\mathrm{W}}}$ is large compared to $M_{\mathrm{W}}$ there are essentially no constraints, whereas if $M_{\overline{\mathrm{W}}} \leqq M_{\mathrm{W}}$ there are restrictions on $M_{\vec{v}}$ which ensure that the decay becomes kinematically forbidden Specifically, if $M_{\tilde{\mathrm{W}}} \leq M_{\mathrm{W}}$ we demand that $M_{\tilde{\nu}_{\tau}}+$ $M_{\tilde{\nu}_{\ell}} \geq M_{\tau}$, but if $M_{\tilde{\mathrm{W}}}>M_{\mathrm{W}}$ lighter $M_{\tilde{\nu}}$ are allowed We feel that the decay $\tau \rightarrow \tilde{\nu}_{\tau} \ell \overline{\tilde{\nu}}_{\ell}$ should be looked for Its effect on the $\tau$ lifetime and the lepton spectrum can be found in ref. [24], note that this decay channel would increase the $\tau$ width and thus decrease the canonical lifetıme. 
(2)

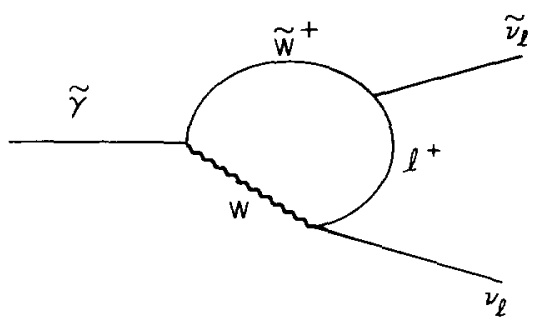

Fig 2 Diagram giving rise to an effective $\tilde{\gamma} \tilde{\nu} \nu$ vertex

If any sneutrino is lighter than $\frac{1}{2}\left(m_{k}-m_{\pi}\right)$ (which admittedly requires repugnant fine tuning in the context of most currently fashionable models) the decay $\mathrm{K}^{+} \rightarrow \pi^{+} \tilde{\nu} \tilde{\bar{\nu}}$ becomes kinematically allowed. Neglectıng phase space, each light sneutrino species contributes $\frac{1}{2}$ of a standard neutrino flavor through the Z-exchange channel, the W \pm “box" contribution is very model dependent.

Also, if $M_{\tilde{\nu}}<M_{\bar{\gamma}}$ as assumed, the photıno will decay through a loop diagram such as shown in fig 2 We can estımate the rate using the full one-loop analysis of Barnett et al [23], although they were calculating with the assumption that $\tilde{\nu} \rightarrow \tilde{\gamma} \nu$, the effective $\tilde{\gamma} \tilde{\nu} \nu$ vertex can be taken from their calculation If we write $: \pi_{\text {eff }}=$ $g_{\text {eff }} \bar{u} P_{\mathrm{R}} u$, then $g_{\text {eff }} \simeq g_{2}^{2} e F / 16 \pi \sqrt{2}$ where $F$ is a function of the various masses, typically of order $\frac{1}{4}$ Then $g_{\text {eff }} \simeq 5 \times 10^{-4}$ e, so $\Gamma(\tilde{\gamma} \rightarrow \tilde{\nu} \bar{\nu}) \simeq g_{\text {eff }}^{2} m_{\hat{\gamma}} / 32 \pi \simeq$ $10^{-7 \frac{1}{2} \alpha m_{\tilde{\gamma}}}$ This gives a lifetıme $\tau_{\tilde{\gamma}} \lesssim 10^{-15} \mathrm{sec}$ for $M_{\tilde{\gamma}}>2 \mathrm{GeV}$ (Ignoring corrections due to $M_{\tilde{v}} \neq 0$ ) Thus for most masses the photino decays very quickly - too rapidly to be observed

Finally, if the photino is unstable, various ways of searching for supersymmetric partners must be re-examined Here we will consider two such categories

(a) Often photinos are assumed to escape detectors, resulting in missing momentum Such analyses are unchanged, since the photino decays into final states $(\tilde{\nu} \bar{\nu})$ which are also invisible

(b) In beam dump experiments photinos are assumed to interact with an interaction cross section which is a few times the neutrino charged current cross section [25] If $\tilde{\gamma} \rightarrow \tilde{\nu} \bar{\nu}$ that situation is somewhat changed Both $\nu$ and $\tilde{v}$ interact, the $\tilde{\nu}$ as in fig 3 Since the Z-exchange contributions to the $\tilde{\nu}$ interaction requires no excitation of heavy squarks or sleptons with an associated kinematical suppression, it will dominate, if a signal is detected. it can be distınguished since it would have a $y$ distribution characteristic of a scalar particle rather than that of a neutrino. Since both $\nu$ and $\tilde{\nu}$ from $\tilde{\gamma}$ decay will interact, there should be a signal Nevertheless previous analyses for gluino production might have to be reinterpreted. For one reason, the $\nu, \tilde{v}$ energies are somewhat degraded by the extra decay, and they may also emerge at large $P_{\mathrm{T}}$ so that fewer of them reach the detector, weakenıng the limit, however, since the FNAL group uses the presence of extra $P_{\mathrm{T}}$ as a possible 
(3)
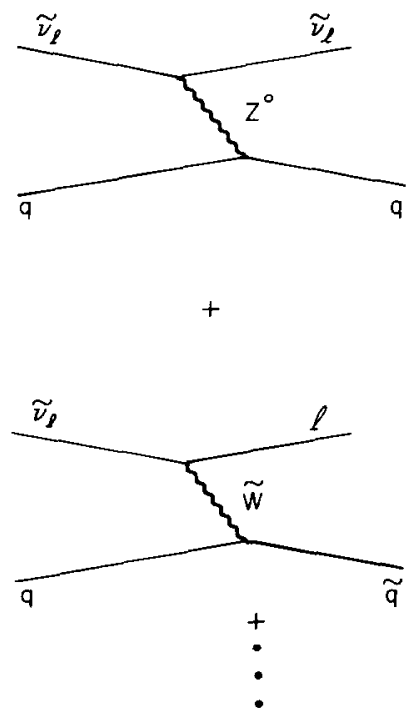

Fig 3 Neutral and charged current sneutrino interactions in a beam dump experiment

signal, this could work either way. Secondly, since the $\nu$ interaction will give both extra charged and extra neutral current events, and the $\tilde{\boldsymbol{\nu}}$ will give some extra charged current events, the decision as to whether or not there are extra events will have to rely on absolute cross sections rather than the comparison of neutral versus charged current cross sections, at present this is very difficult experimentally We conclude that the existing limits on gluino masses may need some modification if sneutrinos are the LSP.

We have seen that it is easy in currently fashonable models for the sneutrino to be the lightest supersymmetric partner We have also shown that a light $\tilde{v}$ is very compatıble with cosmological constraints, and provides a viable candidate for the "dark matter" in galaxies and galactıc clusters. Finally, we have discussed certain interestıng experımental implications of a light $\tilde{\boldsymbol{v}}$ for partıcle physics

\section{Note added}

We have recently received a preprint by L Ibáñez [26] on the same subject. He comes to similar conclusions

We appreciate some enlightenıng discussions with M Claudson, J M Frére and L Hall. We also acknowledge the hospitality of the Aspen Center for Physics where part of this work was done One of us (JSH) would lıke to thank J Ellis for useful conversations related to this work 


\section{References}

[1] P Fayet and S Ferrara, Phys Reports 32C (1977) 249.

P van Nieuwenhuizen, Phys Reports 68 (1981) 189.

J Bagger and J Wess, Supersvmmetrv and supergravty (Princeton Unv Press, 1982),

S J Gates. Jr, M T Grisaru, M Rocek and W Siegel, Superspace (Benjamın/Cummıngs. 1983)

[2] E Witten, Nucl Phys B185 (1981) 513.

M Dine, W Fischler and M Srednickı, Nucl Phys B189 (1981) 575

S Dimopoulos and S Raby, Nucl Phys B192 (1981) 353

[3] $P$ Favet in Unification of the fundamental particle interactions, eds S Ferrara. J Ellis and $P$ van Nieuwenhuizen (Plenum, 1980) p 587,

H E Haber and G L Kane. Phys Reports, to appear

[4] S Weinberg, Phys Rev Lett 48 (1982) 1303.

H Goldberg, Phys Rev Lett 50 (1983) 1419.

L M Krauss, Nucl Phys B227 (1983) 556

[5] J Ellıs, J S Hagelın. D V Nanopoulos, K Olıve and M Srednckı. SLAC-PUB-3171 (1983)

[6] P Fayet, Phys Lett 117B (1982) 460.

J Ellis and J S Hagelin, Phys Lett 122B (1982) 303

[7] J -M Frere and G L Kane, Nucl Phys B223 (1983) 331,

J Ellıs, J S Hagelın, D V Nanopoulos and M Srednickı, SLAC-PUB-3094 (1983)

[8] J Ellis, J -M Frere, J S Hagelın. G L Kane and S T Petcov, SLAC-PUB-3152 (1983)

[9] J Ellis and G G Ross, Phys Lett 117B (1982) 397.

R Arnowitt, A H Chamseddine and P Nath. Phys Rev Lett 50 (1983) 232.

S Weinberg, Phys Rev Lett 50 (1983) 387,

L Alvarez-Gaume, J Polchınskı and M B Wıse, Nucl Phys B221 (1983) 495 ,

L E Ibañez, Nucl Phys B218 (1983) 514

[10] B W Lee and S Weinberg. Phys Rev Lett 39 (1977) 165

[11] K A Olive D Schramm and G Steigman, Nucl Phys B180 (1981) 497

[12] K Freese and D N Schramm. Nucl Phys B233 (1984) 167.

J R Primack and GR Blumenthal, UCSC-TH-162-83, talk fourth work shop on grand unification (Aprll 1983)

[13] P Meszaros, Astron Astrophys 37 (1974) 225

[14] M Axenıdes, R Brandenberger and M Turner. Harvard preprint HUTP-83/A021 (1983)

[15] A Guth and S-Y P1. Phys Rev Lett 49 (1982) 1110. S W Hawking. Phys Lett 115B (1982) 295.

A Starobinskı1, Phys Lett 117B (1982) 175 .

J Bardeen, P Steınhardt and M Turner, Ennico Fermı Institute preprint 83-13

[16] Y Zel'dovich, Mon Not R Astron, Soc 160 (1972) 1,

E Harrison. Phys Rev D1 (1970) 2726

[17] I H Glbert, Astrophys J 144 (1965) 233

[18] P J E Peebles, The large-scale structure of the universe (Prnceton Unuv Press, 1980) p 52

[19] J Ipser and P Sikivie, Phys Rev Lett 50 (1983) 925,

L F Abbott and M B Wise, Nucl Phys B237 (1984) 226

[20] A L Melott et al. Phys Rev Lett 51 (1983) 935

[21] L Alvarez-Gaume, J Polchınskı and M B Wise, ref [9].

J Ellıs, J Hagelın, D V Nanopoulos and K Tamvakıs, SLAC-PUB-3042 (1983),

L E Ibañez and C Lopez, Nucl Phys B233 (1984) 511 ,

LE Ibañez, ref [9]

[22] L E Ibañez and C Lopez, ref [21], table (f)

[23] R M Barnett, HE Haber and K S Lackner, Phys Lett 126B (1983) 64

[24] G L Kane and W B Rolnick, Nucl Phys B217 (1983) 17, B232 (1984) 21

[25] P Fayet, Phys Lett 86B (1979) 272

[26] L E Ibañez, FTUAM-83-28 (1983) 